\title{
Adipophilin and perilipin 3 correlate with total lipid content in
}

\section{human breast milk}

Tereza Pavlova1,2, Zdenek Spacil1, Veronika Vidova ${ }^{1}$, Filip Zlamal1,2, Eliska Cechova ${ }^{1}$, Zuzana Hodicka ${ }^{3}$, Julie Bienertova-Vasku1,2,*

${ }^{1}$ Research Centre for Toxic Compounds in the Environment (RECETOX), Faculty of Science, Masaryk University, Brno, Czech Republic

${ }^{2}$ Department of Pathological Physiology, Faculty of Medicine, Masaryk University, Brno, Czech Republic

${ }^{3}$ Department of Obstetrics and Gynaecology, University Hospital Brno, Brno, Czech Republic

*Correspondence:

Julie Bienertova-Vasku

Research Centre for Toxic Compounds in the Environment (RECETOX), Faculty of Science, Masaryk University, Kamenice 753/5, building A29, 62500 Brno, Czech Republic

Tel. (+420) 549498439

Fax $(+420) 549494340$

Email jbienert@med.muni.cz

Running title: Adipophilin, perilipin 3 and lipid content in breast milk

Keywords: perilipins, milk lipid globules, human breast milk, lipid content, tandem mass spectrometry, ultra-high performance liquid chromatography 
Word count: 4489

\section{Number of figures: 3}

\section{Number of tables: 4}

\section{Role of authors:}

All authors approved the final version of the manuscript.

Tereza Pavlova, M.Sc.: design of the study, generation and interpretation of data, drafting of the manuscript

Zdenek Spacil, Ph.D.: design of the study, mass spectrometry analyses of samples, interpretation of data, revision of the manuscript

Veronika Vidova, M.Sc., Ph.D.: proteomic design and analyses of samples, interpretation of data, revision of the manuscript

Filip Zlamal, M.Sc., Ph.D.: statistic analysis of data, interpretation of data, revision of the manuscript Eliska Cechova, M.Sc., Ph.D.: lipid content of milk determination and interpretation of data, revision of the manuscript

Zuzana Hodicka, M.D., Ph.D.: collection of the samples, interpretation of data, revision of the manuscript Julie Bienertova-Vasku, M.D., Ph.D., Assoc. Prof.: conception and design of the study, interpretation of data, critical revision of the manuscript 


\begin{abstract}
Objective: Lipids are secreted into milk as bilayer-coated structures: milk lipid globules (MLGs). Adipophilin (ADRP) and perilipin 3 (TIP47) are associated with MLGs in human breast milk; however, the role of these proteins in milk lipid secretion is not fully understood. The aim of the study was to investigate levels of ADRP, TIP47 and total lipid content in human breast milk, their mutual correlations and dynamics during lactation.
\end{abstract}

Research Methods \& Procedures: Milk samples from 22 healthy lactating women (Caucasian, Central European) were collected at five time points during lactation $(1-3,12-14,29-30,88-90$ and $178-180$ days postpartum). Mass spectrometry-based method was used for quantification of ADRP and TIP47 in the samples. The gravimetric method was used to determine milk total lipid content.

Results: We observed distinctive trends in ADRP, TIP47 levels and lipid content in human breast milk during the first 6 months of lactation. We also found a significant association between lipid content and ADRP, lipid content and TIP47, and ADRP and TIP47 concentrations in breast milk at all sampling points. Moreover, we derived an equation for estimating the mean lipid content of milk based on ADRP concentration in human breast milk.

Conclusions: A mass spectrometry-based method was developed for quantifying ADRP and TIP47 in human breast milk. Strong mutual correlations were found between ADRP, TIP47 and total lipid content in human breast milk. 


\section{Introduction}

In mammals, milk is secreted during lactation by specialized epithelial cells lining the alveolar lumen of the mammary gland. To satisfy newborns' caloric and nutritional demands, the mammary gland has developed an enormous capacity to synthesize and secrete large quantities of lipids [1]. Milk lipids are synthesized on the endoplasmic reticulum of milk-secreting cells and then accumulated in specialized intracellular organelles called cytosolic lipid droplets (CLDs). In the next stage, CLDs are released by a unique mechanism into milk as structures called milk lipid globules (MLGs).

Throughout evolution, a consistent pathway of lipid secretion has developed based on the ability of eukaryotic cells to synthesize neutral lipids and package them into CLDs. CLDs play a critical role in the regulation of the lipid metabolism and serve as lipid reservoirs for energy generation and membrane synthesis [2]. The neutral lipid core of CLDs, consisting of either triacylglycerols (TAGs) or cholesterol esters, is covered by a phospholipid monolayer with embedded regulatory proteins such as the wellcharacterized perilipin family of proteins [3]. To date, CLDs lacking perilipins have not been identified in mammalian cells [4].

CLDs in milk-secreting cells are secreted by a unique membrane envelopment process [1] resulting in the development of specific structures called MLGs. More specifically, as CLDs in milk-secreting cells move towards the apical membrane of the cells, they are enveloped by their plasma membrane and released as MLGs into the lumen of the mammary gland. In this process, the phospholipid monolayer of CLDs is encased into a bilayer of phospholipids resulting in the three-layered membrane of MLGs known as the milk lipid globule membrane (MLGM). MLGs provide important nutritional and immunological components for newborns and also regulate the rate and site of digestion in newborns' gastrointestinal tracts [5]. The MLG lipid core is primarily composed of TAGs (more than $95 \%$ ) and a small number of partial glycerides, phospholipids, sterols, free fatty acids, etc. [5,6]. MLGM consists of an inner phospholipid monolayer originating from CLD and an outer bilayer membrane which originates from the apical plasma membrane of the milk-secreting cells, composed mainly of cholesterol, phosphatidylcholine and sphingomyelin $[7,8]$. 
Processes regulating milk lipid secretion in humans are far from fully understood. Nevertheless, perilipins are expected to play important role in the milk lipid synthesis and secretion [1]. These evolutionary conserved group of proteins are localized both in the surface of CLDs [9] and MLGs [10,11]. The main function of perilipins is thought to be the regulation of TAG turnover, achieved by controlling lipase access to the neutral lipid core of CLDs [1]. The study aims to establish a quantitative protein assay for perilipins in human breast milk in order to investigate the levels of these proteins in breast milk during lactation. Our objective is to examine the correlation between levels of individual perilipins and total lipid content in human breast milk and to study the dynamics of these levels during the lactation period. The study is mainly focused on two perilipins detected in human breast milk, adipophilin (ADRP) and perilipin 3 (TIP47).

\section{Subjects and Methods}

\section{A. Study subjects and design}

A cohort of 22 healthy lactating women (Caucasian, Central-European) was recruited between October 2009 and June 2010. All women completed a demographic questionnaire. Signed informed consent forms were obtained from all participants and archived. The study was approved by the Committee for Ethics of Medical Experiments on Human subjects, Faculty of Medicine of Masaryk University under the No. 26/2012 (Brno, Czech Republic) in accordance with the Declaration of Helsinki. Inclusion criteria were 1) spontaneous conception, 2) uneventful, singleton pregnancy, 3) spontaneous, uncomplicated delivery, 4) a normal oral 75-g oral glucose tolerance test between 24 and 28 weeks of gestation based on World Health Organization (WHO) criteria [12], 5) appropriate-for-gestational-age neonate, 6) no pre-pregnancy or pregnancy hormonal therapy, previous fertility treatment, surgical or induced delivery, 7) preconception body mass index under $35 \mathrm{~kg} / \mathrm{m}^{2}$ and 8) primiparity.

Milk samples were collected by manual expression from both breasts at several time points, in each case 2 hours after breastfeeding or after a previous manual milk expression. Where possible, a total of $5 \mathrm{ml}$ of milk was collected at all time points: day 1-3 (time point 1), 12-14 (2), 28-30 (3), 88-90 (4) and 178-180 
(5) after birth, i.e. 5 times within the 180 days of the study. Samples were vortexed, distributed into $200 \mu \mathrm{l}$ aliquots and kept frozen at $-80^{\circ} \mathrm{C}$ until further sample processing for analysis. Perilipin concentrations in individual samples were measured and mean and median concentrations at specific time points were calculated. Quality control samples (QC) were prepared by pooling $10 \mu \mathrm{L}$ of milk from each individual sample and subsequently used for the preparation of matrix-matched calibration curve.

\section{B. Chemicals and solvents}

Isotopically labelled proteotypic peptides (SpikeTides ${ }^{\mathrm{TM}} \mathrm{L}$ and Peptides SpikeTides ${ }^{\mathrm{TM}} \mathrm{TQL}$ ) were purchased from JPT Peptide Technologies (Germany) and used to detect and quantify selected perilipins. MS grade Trypsin Gold (Promega) was used for enzymatic protein digestion. Solid phase extraction (SPE) was performed to desalt and purify peptides using Bond Elut C18 200 mg (Agilent). Ammonium bicarbonate buffer (200 mmol/L, $\mathrm{pH}=8$, Sigma Aldrich) was added to breast milk samples to adjust $\mathrm{pH}$ for tryptic digestion; LC-MS grade formic acid (FA, Sigma Aldrich) was added after digestion to acidify samples for loading on SPE and 0.1\% FA was also used as a UHPLC mobile phase additive. Organic solvents for mobile phase, sample processing and dilution of stock solutions i.e. LC-MS grade methanol and acetonitrile were purchased from Avantor. Ammonia (Lach-Ner), ethanol (Merck), diethyl ether (Promochem) and n-hexane (Lach-Ner) were used for lipid extraction. Ultrapure water (resistivity of 18.2 $\mathrm{M} \Omega . \mathrm{cm}$ at $25^{\circ} \mathrm{C}$ ) was obtained using the Millipore purification system (Simplicity 185 system, Millipore Corp.).

\section{Total lipid content of milk}

The liquid-liquid extraction Röse-Gottlieb reference method used to determine the total lipid content of milk (23) was modified in order to accommodate lower sample amounts compared to the original method protocol. Milk samples were refrigerated overnight to thaw and then sonicated for $20 \mathrm{~min} .5 \mathrm{~mL}$ of each milk sample was mixed with $750 \mu \mathrm{L}$ of ammonia in water $(25 \%), 5 \mathrm{~mL}$ of ethanol, $12.5 \mathrm{~mL}$ of diethyl ether and $12.5 \mathrm{~mL}$ of $\mathrm{n}$-hexane and shaken vigorously once each solvent was added. Samples were centrifuged and the upper phase was collected into a weighed vial. Diethyl-ether and n-hexane were then added again to the lower phase, mixed and centrifuged; the collected upper layer (i.e. the second 
fraction) was combined with the first fraction. Finally, a third fraction was collected in the same manner. The solvent was evaporated from the combined fraction under a stream of nitrogen and the total lipid weight was gravimetrically determined.

\section{Protein concentrations in milk}

\section{Protein assay design}

Crude stable-isotopically labelled proteotypic peptides (SpikeTides ${ }^{\mathrm{TM}} \mathrm{L}$ ) (Table 1) were selected for four perilipins (perilipin 1, ADRP, TIP47 and perilipin 5) according to the UniProt database (www.uniprot.org) and SRMAtlas repository (www.srmatlas.org) and used to generate a transition list for selected reaction monitoring (SRM) as previously described [13]. Stable-isotopically labelled peptides with trypsin cleavable tags (SpikeTides ${ }^{\mathrm{TM}} \mathrm{TQL}$ ) of certified exact concentrations were used for the absolute quantification of ADRP and TIP47 in breast milk.

\begin{tabular}{llll}
\hline Protein & Gene & Peptide sequence & SIL peptideSpikeTides $^{\text {TM }}$ \\
\hline ADRP & PLIN2 & SELLVEQYLPLTEEELEK & L and TQL \\
ADRP & PLIN2 & TITSVAMTSALPIIQK & L and TQL \\
ADRP & PLIN2 & SQQTISQLHSTVHLIEFAR & $\mathrm{L}$ \\
ADRP & PLIN2 & DSVASTITGVMDK & L and TQL \\
ADRP & PLIN2 & EVSDSLLTSSK & L and TQL \\
Perilipin 1 & PLIN1 & LASGGADLALGSIEK & $\mathrm{L}$ \\
Perilipin 1 & PLIN1 & VLHLTPAPAVSSTK & $\mathrm{L}$ \\
TIP47 & PLIN3 & IATSLDGFDVASVQQQR & $\mathrm{L}$ and TQL \\
Perilipin 5 & PLIN5 & SVSHAVDVVLEK & $\mathrm{L}$ \\
Perilipin 5 & PLIN5 & SVDALQTAFADAR & $\mathrm{L}$ \\
\hline
\end{tabular}

\section{Table 1: Crude stable-isotopically labelled proteotypic peptides for perilipins}

The listed SpikeTides ${ }^{T M} L$ peptides $(L)$ were used to screen for four perilipins in breast milk and to generate a transition list. Perilipins with concentrations above the detection limit in breast milk were quantified in milk using stable-isotopically labeled (SIL) peptides with trypsin cleavable tags (TQL) with the same sequence as SpikeTides ${ }^{T M} L$. 


\section{Quantitative method optimization}

Matrix-matched (MM) calibration was prepared using QC samples and a mix of TQL peptides at 6 concentration levels within a range of 5-400 nM (Supplemental Figure 1, Supplemental Table 1). Limit of detection (LOD) and limit of quantification (LOQ) values were calculated from the MM calibration curve using the lowest concentration level with a coefficient of variance $<20 \%(n=6)$ to determine standard deviation (SD), e.g. $3^{*}$ SD divided by SLOPE (for LOD) and $10^{*}$ SD divided by SLOPE (for LOQ) [13]. Linearity within the $5-400 \mathrm{nM}$ concentration range was $\mathrm{R}^{2}>0.99$.

Enzymatic digestion duration (8, 16 and $24 \mathrm{~h}$ ) was optimized and $16 \mathrm{~h}$ trypsin digestion was determined as optimal based on the highest response of light and heavy peptides compared to the remaining reaction times (Supplemental Figure 2).

\section{Sample preparation}

$10 \mu \mathrm{L}$ of ammonium bicarbonate buffer $(200 \mathrm{mM}, \mathrm{pH}=8), 10 \mu \mathrm{L}$ of TQL peptides standard solution and 3 $\mu \mathrm{L}$ of trypsin $(1 \mu \mathrm{g} / \mu \mathrm{L})$ were added to $30 \mu \mathrm{L}$ of breast milk, followed by incubation at $37^{\circ} \mathrm{C}$. Individual TQL peptide concentrations in the standard solution were as follows: SELLVEQYLPLTEEELK (ADRP), 1200 nmol/L; DSVASTITGVMDK (ADRP), 750 nmol/L; $\quad$ EVSDSLLTSS $\quad$ (ADRP), $750 \quad \mathrm{nmol} / \mathrm{L}$; DTVATQLSEAVDATR (TIP47), 300 nmol/L; IATSLDGFDVASVQQQR, $150 \mathrm{nmol} / \mathrm{L}$. Trypsin digestion was terminated after 16 hours by adding $300 \mu \mathrm{L}$ of $2 \%$ FA in water. SPE cartridges were conditioned as follows: $1 \mathrm{~mL}$ of methanol, $1 \mathrm{~mL}$ of $50 \%$ acetonitrile (with $2 \% \mathrm{FA}$ ) and $1 \mathrm{~mL}$ of $2 \% \mathrm{FA}$. The sample was loaded into a cartridge, washed with $2 \mathrm{~mL}$ of $2 \%$ FA and eluted with $1 \mathrm{~mL}$ of $50 \%$ acetonitrile (with $2 \%$ FA). The sample solvent was removed under a stream of nitrogen and the sample was re-dissolved in 30 $\mu \mathrm{L}$ of $5 \%$ acetonitrile, with final peptide concentrations as follows: $400,250,250,100$ and $50 \mathrm{nmol} / \mathrm{L}$ respectively.

\section{Protein quantification - UHLPC-MS/MS (SRM)}

Samples were analyzed using ultra-high performance liquid chromatography (UHPLC) and tandem mass spectrometry (MS/MS), specifically by selected reaction monitoring (SRM). The UHPLC system (1290 
Infinity II) and triple quadrupole (QqQ) mass analyzer (model 6495) were utilized (Agilent Technologies, CA, USA). Reversed-phase UHPLC separation utilized analytical column C18 CSH column (1.7 $\mu \mathrm{m}$, $2.1 \times 100 \mathrm{~mm}$, Waters). MS/MS conditions were optimized using Optimizer software (Agilent Technologies). UHPLC separation was performed with water (A) and acetonitrile (B), both with an addition of $0.1 \% \mathrm{FA}$ at $0.3 \mathrm{~mL} / \mathrm{min}$ using $35 \mathrm{~min}$ analytical gradient $(0.00 \mathrm{~min} 5 \% \mathrm{~B}, 25.00 \mathrm{~min} 30 \% \mathrm{~B}$, $25.30 \min 95 \%$ B, 30.00 min $95 \%$ B, 31.00 min 5 \% B, 35.00 min 5 \% B). Milk samples were analyzed in positive ion and dynamic SRM mode with a 3.5 min retention time window. The assay library is specified in supplementary materials (Supplemental Table 2). Five SRM transitions were used as qualifiers and the most intense signal was used as a quantifier. The quantifiers are marked in bold in Supplemental Table 2.

\section{E. Statistical analyses}

All statistical analyses were carried out using statistical software R (version 3.3.3). Conventional values of $p<0.05$ were considered statistically significant. Variables were characterized by mean, standard deviation, minimum and maximum values. The Pearson correlation coefficient $(r)$ was used to assess the strength of associations between variables. First, the linear mixed-effects model fit by restricted maximum likelihood (REML) estimation was used to find differences between distinct time points followed by $p$ value adjustment using the Tukey method. Second, the same type of modelling was utilized to locate significant trends in given variable levels over time.

\section{Results}

\section{A. ADRP and TIP47 concentrations and total lipid content in breast milk}

The baseline characteristics of study subjects are summarized in Table 2. The table includes ADRP and TIP47 concentrations and the total lipid content of breast milk established at five time points during the lactation period. ADRP and TIP47 levels and total lipid content were measured in each individual sample at each time point; the results are visualized in a notched-box plot (Figure 1). We found no significant differences between ADRP breast milk concentrations at individual time points during lactation. However, TIP47 concentration in breast milk was found to be significantly increased at all time points compared to 
time point $1(p<0.001)$. Compared to time point 1 the lipid content of milk was significantly increased at time point $2(p=0.019)$ and at time point $5(p<0.001)$ (Figure 1$)$.

\begin{tabular}{llllll}
\hline & Mean & Median & SD & Min & Max \\
\hline Maternal age [years] & 29.9 & 30.4 & 3.3 & 7.9 & 40.2 \\
Preconception BMI [kg/m²] & 21.4 & 21.5 & 7.0 & 17.7 & 33.4 \\
BMI at delivery [kg/m²] & 27.7 & 26.8 & 3.7 & 22.0 & 38.9 \\
Pregnancy weight gain [kg] & 15.2 & 15.0 & 4.2 & 8.0 & 25.0 \\
Birth length [cm] & 49.9 & 50.0 & 1.6 & 47.0 & 52.0 \\
Birth weight [kg] & 3.2 & 3.3 & 0.6 & 2.2 & 4.5 \\
Adipophilin 1-3 days postpartum [ng/mL] & 237.4 & 175.1 & 143.2 & 80.7 & 541.3 \\
Adipophilin 12-14 days postpartum [ng/mL] & 261.5 & 254.9 & 88.2 & 65.4 & 65.4 \\
Adipophilin 28-30 days postpartum [ng/mL] & 255.0 & 245.3 & 89.6 & 104.7 & 465.3 \\
Adipophilin 88-90 days postpartum [ng/mL] & 254.8 & 198.5 & 172.1 & 29.8 & 326.6 \\
Adipophilin 178-180 days postpartum [ng/mL] & 259.5 & 259.9 & 69.6 & 156.0 & 351.4 \\
TIP47 1-3 days postpartum [ng/mL] & 31.0 & 26.1 & 22.1 & 1.3 & 89.6 \\
TIP47 12-14 days postpartum $[\mathrm{ng} / \mathrm{mL}]$ & 95.3 & 88.9 & 35.1 & 26.3 & 174.6 \\
TIP47 28-30 days postpartum $[\mathrm{ng} / \mathrm{mL}]$ & 89.8 & 90.3 & 28.7 & 26.3 & 174.6 \\
TIP47 88-90 days postpartum $[\mathrm{ng} / \mathrm{mL}]$ & 83.5 & 73.3 & 73.9 & 29.8 & 326.6 \\
TIP47 178-180 days postpartum [ng/mL] & 78.3 & 71.5 & 23.4 & 47.9 & 125.3 \\
Lipid content 1-3 days postpartum [ng/mL] & 19.8 & 15.7 & 11.6 & 7.9 & 40.2 \\
Lipid content 12-14 days postpartum [ng/mL] & 34.7 & 35.5 & 12.8 & 4.9 & 64.1 \\
Lipid content 28-30 days postpartum [ng/mL] & 37.0 & 31.2 & 15.2 & 14.4 & 70.9 \\
Lipid content 88-90 days postpartum [ng/mL] & 38.3 & 31.8 & 24.8 & 9.8 & 94.5 \\
Lipid content 178-180 days postpartum [ng/mL] & 55.3 & 52.3 & 22.0 & 20.1 & 84.5 \\
\hline
\end{tabular}

Table 2: Anthropometric data, ADRP and TIP47 concentrations and lipid content in breast milk $S D$, standard deviation 

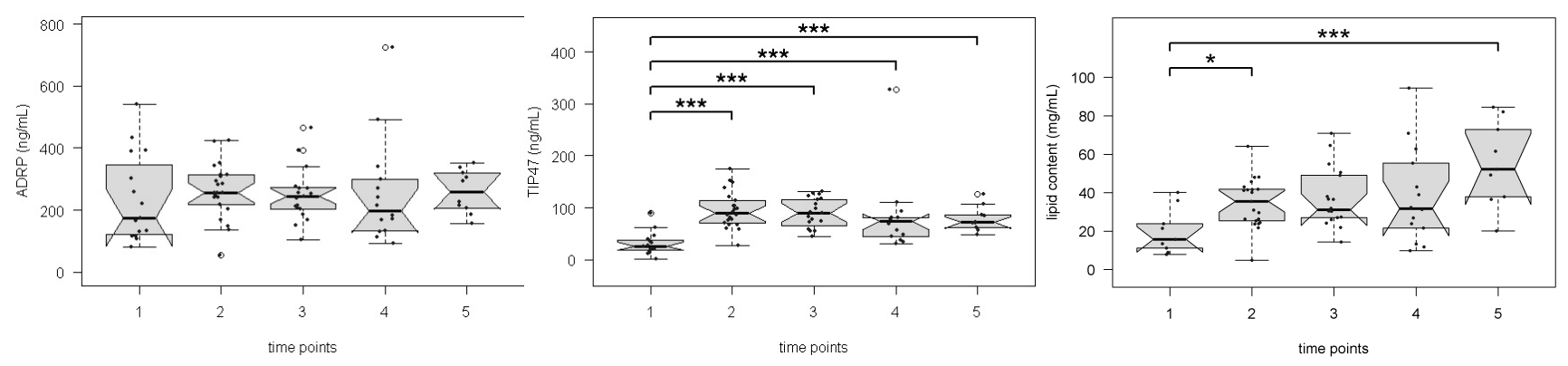

Figure 1: Levels of ADRP, TIP47 and lipid content of breast milk

Notched-box-plot of ADRP, TIP47 and lipid concentrations in human breast milk during lactation. The asterisks represent significant differences $\left({ }^{*}, 0.01<p \leq 0.05 ;{ }^{* *}, 0.001<p \leq 0.01 ;{ }^{* * *}, p \leq 0.001\right)$ in concentrations between time points (time point 1, 1-3 days postpartum; time point 2, 12-14 days postpartum; time point 3, 28-30 days postpartum; time point 4, 88-90 days postpartum; time point 5, 178-180 days postpartum).

We used a linear mixed-effects model fit by REML to evaluate the trend in ADRP/TIP47/lipid content dynamics during lactation. We observed a linear dependence of ADRP concentration on time, specifically a constant ADRP level throughout the lactation period (Figure 2). On the other hand, we also noted the non-linear dependence of TIP47 and lipid content on time, suggesting a cubic polynomial regression (TIP 47: $\beta(1)=4.416, p<0.001 ; \beta(2)=-1.365, p<0.001 ; \beta(3)=0.131, p<0.001$ and lipid content: $\beta(1)=$ 2.445, $p=0.005 ; \beta(2)=-0.804, p=0.012 ; \beta(3)=0.131, p=0.085)$, where $\beta(k)$ represents coefficient at $\mathrm{k}^{\text {th }}$ power of time (Figure 2). 

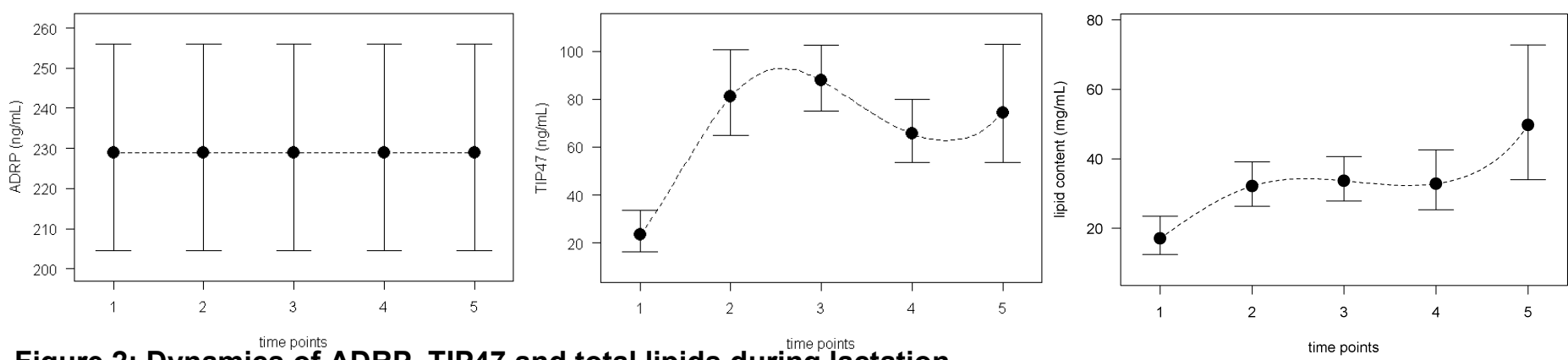

Figure 2: Dynamics of ADRP, TIP47 and total lipids during points lactation

The linear dependence of ADRP and cubic polynomial dependence of TIP47 and total lipids on time. The dashed line represents the predicted trend of ADRP, TIP47 and lipid concentrations during lactation based on a linear mixed-effects model. The black dots represent the estimated mean values of each variable with a $95 \%$ confidence interval.

\section{B. Correlation}

We found significant correlations between total lipid content and ADRP, between lipid content and TIP47 and also between ADRP and TIP47 at all lactation time points. The only exception is the relationship between the lipid content of milk and TIP47 concentration at time point 1 where the correlation was found to be only borderline significant (Table 3).

\begin{tabular}{llllllllllllll}
\hline Time & \multicolumn{3}{c}{ Lipid content vs ADRP } & \multicolumn{1}{c}{ Lipid content vs TIP47 } & \multicolumn{4}{c}{ ADRP vs TIP47 } \\
point & $\mathrm{n}$ & $\mathrm{r}$ & $95 \% \mathrm{Cl}$ & $\mathrm{p}$ & $\mathrm{n}$ & $\mathrm{r}$ & $95 \% \mathrm{Cl}$ & $\mathrm{p}$ & $\mathrm{n}$ & $\mathrm{r}$ & $95 \% \mathrm{Cl}$ & $\mathrm{p}$ \\
\hline 1 & 6 & 0.86 & $(0.44 ; 0.97)$ & $\mathbf{0 . 0 0 4}$ & 6 & 0.63 & $(-0.09 ; 0.92)$ & 0.079 & 15 & 0.75 & $(0.13 ; 0.95)$ & $<0.001$ \\
2 & 21 & 0.89 & $(0.76 ; 0.95)$ & $<0.001$ & 21 & 0.77 & $(0.53 ; 0.90)$ & $<0.001$ & 22 & 0.85 & $(0.67 ; 0.93)$ & $<0.001$ \\
3 & 18 & 0.88 & $(0.72 ; 0.95)$ & $<0.001$ & 18 & 0.80 & $(0.56 ; 0.92)$ & $<0.001$ & 19 & 0.86 & $(0.68 ; 0.94)$ & $<0.001$ \\
4 & 14 & 0.79 & $(0.48 ; 0.92)$ & $<0.001$ & 14 & 0.76 & $(0.44 ; 0.91)$ & $<0.001$ & 14 & 0.87 & $(0.67 ; 0.95)$ & $<0.001$ \\
5 & 9 & 0.80 & $(0.40 ; 0.94)$ & $\mathbf{0 . 0 0 2}$ & 9 & 0.82 & $(0.45 ; 0.95)$ & $\mathbf{0 . 0 0 1}$ & 10 & 0.72 & $(0.23 ; 0.92)$ & $\mathbf{0 . 0 0 8}$ \\
\hline
\end{tabular}

Table 3: Correlations between ADRP, TIP47 and lipid content of human breast milk at different time points during lactation

$n$, number of mothers sampled at a given time point; $r$, Pearson correlation coefficient; Cl, confidence interval; time point 1, 1-3 days postpartum; time point 2, 12-14 days postpartum; time point 3, 28-30 days postpartum; time point 4, 88-90 days postpartum; time point 5, 178-180 days postpartum 


\section{Confounding factors}

Based on a partial correlation we found ADRP to play the role of a confounding factor in the relationship between ADRP, TIP47 and the lipid content of milk. The results suggest a gradual weakening of the effect from time point 1 to time point 3 and its subsequent disappearance by time points 4 and 5 . The effect is expressed as a change of the Pearson correlation coefficient ( $r$ ) once the effect of the third variable is removed (partial r) (Table 4).

\begin{tabular}{lllllll}
\hline \multirow{2}{*}{ Time point } & \multicolumn{2}{l}{ Lipid content vs ADRP } & \multicolumn{2}{l}{ Lipid content vs TIP47 } & \multicolumn{2}{l}{ ADRP vs TIP47 } \\
\cline { 2 - 7 } & $r$ & partial $r$ & $r$ & partial $r$ & $r$ & partial $r$ \\
\hline 1 & 0.86 & 0.76 & 0.63 & -0.05 & 0.75 & 0.52 \\
2 & 0.89 & 0.71 & 0.77 & 0.06 & 0.85 & 0.56 \\
3 & 0.88 & 0.62 & 0.80 & 0.17 & 0.86 & 0.55 \\
4 & 0.79 & 0.38 & 0.76 & 0.26 & 0.87 & 0.69 \\
5 & 0.80 & 0.53 & 0.82 & 0.59 & 0.72 & 0.19 \\
\hline
\end{tabular}

Table 4: Partial correlation between ADRP, TIP47 and total lipid content of breast milk

$r$, Pearson correlation coefficient; partial $r$, Pearson correlation coefficient following the removal of the effects of the third variable; postpartum time point 1, 1-3 days; time point 2, 12-14 days; time point 3, 2830 days; time point 4, 88-90 days; time point 5, 178-180 days

\section{Prediction of lipid content in milk based on ADRP}

Based on the linear mixed-effects model fit by REML we derived a formula for the mean lipid content of milk:

$\log ($ lipid $)=-2.35+0.99 \times \log (A D R P)+0.15 \times($ time point $)$

where lipid is the lipid content of milk $(\mathrm{mg} / \mathrm{mL}), A D R P$ is adipophilin concentration in milk $(\mu \mathrm{g} / \mathrm{mL})$ and time point is $1,2,3,4$ or 5 based on the day of sampling $(1-3,12-14,28-30,88-90$ or $178-180)$. Lipid content in milk may also be derived, separately for each time point, from graphs included in Figure 3. 

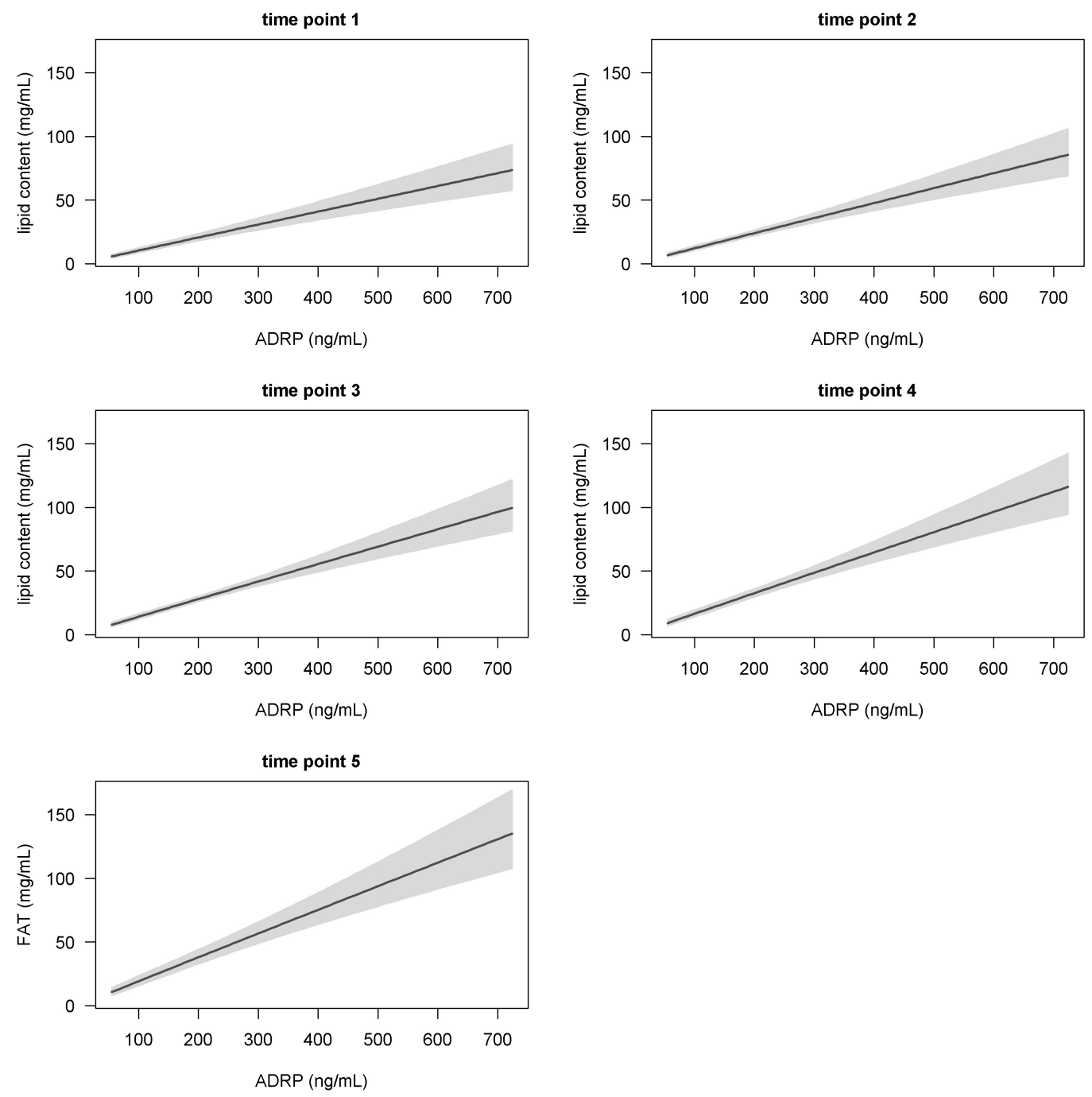

Figure 3: Prediction of lipid content in milk with a $95 \%$ confidence interval

Due to the strong correlation between ADRP levels and the lipid content of milk, it is possible to predict one using the other at individual time points using these graphs. The line represents the predicted mean value. The grey area represents a $95 \%$ confidence interval for the mean.

\section{Discussion}

Lipids in human breast milk constitute a major source of energy, essential nutrients and vitamins for the infant. They ensure appropriate neonatal growth and development, support immune system function and help synthesize bioactive lipid signaling molecules and cell membranes. While the volume of findings on 
milk lipid formation is growing, the number of unanswered questions is increasing at the same time [1]. An area which remains insufficiently understood is the molecular mechanisms of milk lipid secretion. Evolutionarily ancient perilipins include five members with considerable sequence similarities - perilipin 1 (encoded by the PLIN1 gene), perilipin 2/adipophilin (PLIN2), perilipin 3 (PLIN3), perilipin 4 (PLIN4), and perilipin 5 (PLIN5). A growing body of evidence suggests that the main functions of all perilipins include CLD formation and stabilization under various conditions [14]. Perilipins are either exclusively associated with CLD (PLIN1, 2) or exist as exchangeable proteins stable in both the cytoplasm and CLDs (PLIN3, 4, 5) [4]. Moreover, the perilipin profiles of CLDs differ based on cell source [14], neutral lipid composition [4] and size $[4,15]$. To contribute to the existing understanding of molecular frameworks regulating milk lipid synthesis and secretion, this study thus investigated selected perilipins involved in the process of milk lipid secretion.

We initially selected specific peptide standards (proteotypic peptides) representing four perilipins (perilipin 1, ADRP, TIP47 and perilipin 5). We screened all four perilipin proteins in human breast milk, detecting ADRP and TIP47 in concentrations above detection limit. We did not detect perilipin 1 and 5 . While perilipin 1 is presumably expressed in the mammary gland and localized exclusively in CLDs [16], it was previously not detected in milk human breast milk [17]. Perilipin 5 has never been associated with MLGs or breast milk. If trace amounts of perilipin 1 and 5 are indeed present in human breast milk, their concentrations were below the detection limit of the described SRM protein assay. This study further focused on quantifying ADRP and TIP47 in human breast milk and investigating their relation to the lipid content of breast milk.

\section{A. Adipophilin}

Adipophilin is among the most abundant proteins found in both CLDs and MLGs. It has been hypothesized to be a key player in milk lipid formation and secretion [2]. Adipophilin was originally identified in the teratoma-derived adipogenic cell line (1246 Cells) as an mRNA molecule expressed early during adipocyte differentiation [18]. The $50 \mathrm{kDa}$ protein was thus named adipose differentiation-related protein (ADRP) [19]. ADRP expression is not limited to adipocytes but is ubiquitous, in contrast to perilipins 1, 4 and 5, which exhibit more limited tissue expression [4]. ADRP transcript levels in milk- 
secreting cells are significantly greater than in other cell types, including adipocytes. Overall, it seems that the increased expression of adipophilin is unique to milk-secreting cells [17]. Although ADRP levels have been reported to correlate with lipid accumulation in a variety of cells and tissues [19-21], to the best of our knowledge this is the first study to show a correlation between ADRP and the lipid content of human breast milk.

The detection of ADRP in breast milk began with its identification in human, bovine and rat MLGs as a major component of the globule surface membrane [22]. Later, ADRP was colocalized with xanthine oxidoreductase and buyrophilin at the apical plasma membrane of both mice secretory epithelial cells in mammary gland and MLGs [23]. In the mouse mammary gland, these three proteins form a stable complex which probably acts as a functional link coupling CLDs and the apical site membrane of secretory epithelial cells in the mammary gland during the membrane envelopment process of CLDs. CLDs coated with the apical plasma membrane are then released into the lumina of mammary alveoli as MLGs. Nevertheless, the factors regulating milk lipid secretion remain poorly defined.

Only one previous study reports on the quantification of ADRP in human breast milk [24] utilizing the immunoanalytical ELISA technique. ADRP was quantified in human breast milk during a 12-month lactation period [24]. ADRP concentrations measured by ELISA were approximately one order of magnitude higher compared to our results measured by SRM protein assay. Specifically, at time point 1 we measured mean protein concentrations of $0.24 \pm 0.14 \mu \mathrm{g} / \mathrm{mL}$ compared to $1.98 \pm 0.12 \mu \mathrm{g} / \mathrm{mL}$ determined by Mitrova et al. (2014), at time point $30.26 \pm 0.09 \mu \mathrm{g} / \mathrm{mL}$ vs $2.83 \pm 0.21 \mu \mathrm{g} / \mathrm{mL}$, at time point $40.25 \pm 0.17 \mu \mathrm{g} / \mathrm{mL}$ vs $2.39 \pm 0.17 \mu \mathrm{g} / \mathrm{mL}$ and at time point $50.26 \pm 0.07 \mathrm{ng} / \mathrm{mL}$ vs $2.57 \pm 0.16 \mu \mathrm{g} / \mathrm{mL}$. The disparity may be due to sample preparation, as Mitrova et al. used skimmed milk in comparison to whole milk utilized in the present study. However, we believe that the removal of the fatty layer from the milk samples would rather result in underestimated level of the MLGM protein. The observed disparity may also be due to different selectivity of both techniques i.e. potential cross-reactivity of antibody utilized for immunoaffinity enrichment in ELISA technique as compared to absolute sequence specificity of SRM determination by tandem mass spectrometry (MS/MS) [25]. 


\section{B. Perilipin 3}

Like ADRP, TIP47 is also expressed in the mammary gland [16] and has been associated with MLGs in human breast milk [10]. TIP47 was originally identified as a $48 \mathrm{kDa}$ placental protein PP17 [26]. In contrast to ADRP, the association of TIP47 with CLDs is controversial. While TIP47 has been reported to be associated with intracellular CLDs $[10,27,28]$, it is not associated with CLDs in milk-secreting cells but is diffusely distributed in the milk-secreting cell cytoplasm; furthermore, its expression patterns are not correlated with CLD accumulation [16]. TIP47 has been detected in a fat-depleted fraction but not in MLGs in mouse milk; its expression has been found to decrease during mammary gland development [17]. On the other hand, TIP47 has been detected in human breast milk MLGs $[10,29]$. Thus, both the localization and the biological role of TIP47 in breast milk remain unclear. By comparison, ADRP in secretory epithelial cells is associated exclusively with CLDs. The different localization of ADRP in secretory cells is consistent with our results, which show different dynamics during lactation and indicate a relationship to the lipid content of milk. While ADRP looks like a confounder in the relationship between ADRP, TIP47 and lipid content, we suggest that the correlation between TIP47 and total lipid content is dependent on the association between ADRP and TIP47.

\section{Lipid content of milk}

Information about the total lipid content in breast milk is important e.g. with respect to sufficient milk lipid production needed for appropriate neonatal growth and development or in order to normalize levels of non-polar organic pollutants in human breast milk such as persistent pesticides or polychlorinated biphenyls [30]. However, original referenced method for the determination of the lipid content of breast milk required a relatively large volume of breast milk and the use of highly flammable solvents; moreover, the process was laborious, time-consuming and highly prone to error [31]. On the other hand, the formula presented in our study constitutes an alternative method for determining the total lipid content of breast milk based on ADRP concentration. The newly developed ADRP protein quantification assay requires only $30 \mu \mathrm{L}$ of sample and is accurate, reproducible and suitable method for routine clinical laboratory. 
Our hypothesis about the correlation between the lipid content of milk and adipophilin is supported by the fact that ADRP colocalizes with CLDs secreted by mammary secretory epithelial cells into milk [17]. Furthermore, it is also one of the most abundant proteins found in MLGs [11]. In this study we have discovered the correlation between the lipid content of milk and ADRP levels and thus confirmed the hypothesis. The finding suggest that ADPR levels at specific time points during lactation are predictive of the total lipid content in breast milk at investigated time points within lactation period.

\section{Conclusion}

We have established a UHPLC-MS/MS assay for the absolute quantification of ADRP and TIP47 in human breast milk. We found strong correlations between ADRP and lipid content, TIP47 and lipid content and also between ADRP and TIP47 in human breast milk.

\section{Acknowledgements}

This study was carried out in part thanks to funds provided by the RECETOX research infrastructure (Ministry of Education, Youth and Sports - MEYS, LM2015051), CETOCOEN PLUS (MEYS, CZ.02.1.01/0.0/0.0/15_003/0000469) and the Czech Science Foundation (project No. 17-24592Y). Many thanks go to the participating mothers as well as to the medical staff of University Hospital Brno and the entire study team. 


\section{References}

[1] McManaman JL. Formation of milk lipids: a molecular perspective. Clin Lipidol 2009;4:391-401. doi:10.2217/clp.09.15.

[2] Walther TC, Farese RV. Lipid Droplets And Cellular Lipid Metabolism. Annu Rev Biochem 2012;81:687-714. doi:10.1146/annurev-biochem-061009-102430.

[3] Brasaemle DL. Thematic review series: Adipocyte Biology. The perilipin family of structural lipid droplet proteins: stabilization of lipid droplets and control of lipolysis. J Lipid Res 2007;48:2547-59. doi:10.1194/jlr.R700014-JLR200.

[4] Sztalryd C, Brasaemle DL. The perilipin family of lipid droplet proteins: Gatekeepers of intracellular lipolysis. Biochim Biophys Acta BBA - Mol Cell Biol Lipids 2017;1862:1221-32. doi:10.1016/j.bbalip.2017.07.009.

[5] Garcia C, Innis S. Structure of the human milk fat globule. Lipid Technol 2013;25:223-6. doi:10.1002/lite.201300303.

[6] Patton S, Jensen RG. Lipid metabolism and membrane functions of the mammary gland. Prog Chem Fats Other Lipids 1975;14:163-277. doi:10.1016/0079-6832(75)90004-X.

[7] Heid HW, Keenan TW. Intracellular origin and secretion of milk fat globules. Eur J Cell Biol 2005;84:245-58. doi:10.1016/j.ejcb.2004.12.002.

[8] German JB, Dillard CJ. Composition, Structure and Absorption of Milk Lipids: A Source of Energy, Fat-Soluble Nutrients and Bioactive Molecules. Crit Rev Food Sci Nutr 2006;46:57-92. doi:10.1080/10408690590957098.

[9] Ducharme NA, Bickel PE. Lipid droplets in lipogenesis and lipolysis. Endocrinology 2008;149:9429. doi:10.1210/en.2007-1713.

[10] Than NG, Sumegi B, Bellyei S, Berki T, Szekeres G, Janaky T, et al. Lipid droplet and milk lipid globule membrane associated placental protein 17b (PP17b) is involved in apoptotic and differentiation processes of human epithelial cervical carcinoma cells. Eur J Biochem 2003;270:1176-88. doi:10.1046/j.1432-1033.2003.03475.x.

[11] Chong BM, Reigan P, Mayle-Combs KD, Orlicky DJ, McManaman JL. Determinants of adipophilin function in milk lipid formation and secretion. Trends Endocrinol Metab TEM 2011;22:211-7. doi:10.1016/j.tem.2011.04.003.

[12] Prevention of diabetes mellitus. Report of a WHO Study Group. World Health Organ Tech Rep Ser 1994;844:1-100.

[13] Vidova V, Spacil Z. A review on mass spectrometry-based quantitative proteomics: Targeted and data independent acquisition. Anal Chim Acta 2017;964:7-23. doi:10.1016/j.aca.2017.01.059.

[14] Itabe H, Yamaguchi T, Nimura S, Sasabe N. Perilipins: a diversity of intracellular lipid droplet proteins. Lipids Health Dis 2017;16:83. doi:10.1186/s12944-017-0473-y.

[15] Lu J, Argov-Argaman N, Anggrek J, Boeren S, Hooijdonk T van, Vervoort J, et al. The protein and lipid composition of the membrane of milk fat globules depends on their size. J Dairy Sci 2016;99:4726-38. doi:10.3168/jds.2015-10375.

[16] Russell TD, Palmer CA, Orlicky DJ, Bales ES, Chang BH-J, Chan L, et al. Mammary glands of adipophilin-null mice produce an amino-terminally truncated form of adipophilin that mediates milk lipid droplet formation and secretion. J Lipid Res 2008;49:206-16. doi:10.1194/jlr.M700396-JLR200.

[17] Russell TD, Palmer CA, Orlicky DJ, Fischer A, Rudolph MC, Neville MC, et al. Cytoplasmic lipid droplet accumulation in developing mammary epithelial cells: roles of adipophilin and lipid metabolism. J Lipid Res 2007;48:1463-75. doi:10.1194/jlr.M600474-JLR200.

[18] Jiang HP, Harris SE, Serrero G. Molecular cloning of a differentiation-related mRNA in the adipogenic cell line 1246. Cell Growth Differ Mol Biol J Am Assoc Cancer Res 1992;3:21-30.

[19] Jiang HP, Serrero G. Isolation and characterization of a full-length cDNA coding for an adipose differentiation-related protein. Proc Natl Acad Sci U S A 1992;89:7856-60.

[20] Steiner S, Wahl D, Mangold BL, Robison R, Raymackers J, Meheus L, et al. Induction of the adipose differentiation-related protein in liver of etomoxir-treated rats. Biochem Biophys Res Commun 1996;218:777-82. doi:10.1006/bbrc.1996.0138. 
[21] Buechler C, Ritter M, Duong CQ, Orso E, Kapinsky M, Schmitz G. Adipophilin is a sensitive marker for lipid loading in human blood monocytes. Biochim Biophys Acta 2001;1532:97-104.

[22] Heid HW, Schnölzer M, Keenan TW. Adipocyte differentiation-related protein is secreted into milk as a constituent of milk lipid globule membrane. Biochem J 1996;320:1025-30.

[23] McManaman JL, Palmer CA, Wright RM, Neville MC. Functional regulation of xanthine oxidoreductase expression and localization in the mouse mammary gland: evidence of a role in lipid secretion. J Physiol 2002;545:567-79.

[24] Mitrova K, Karpisek M, Durilova M, Dragusin LG, Nevoral J, Bronsky J. Development of highsensitive ELISA method for detection of adipophilin levels in human colostrum and breast milk. J Clin Lab Anal 2014;28:255-60. doi:10.1002/jcla.21675.

[25] Baker M. Reproducibility crisis: Blame it on the antibodies. Nature 2015;521:274-6. doi:10.1038/521274a.

[26] Bohn H, Kraus W, Winckler W. Purification and characterization of two new soluble placental tissue proteins (PP13 and PP17). Oncodevelopmental Biol Med J Int Soc Oncodevelopmental Biol Med 1983;4:343-50.

[27] Miura S, Gan J-W, Brzostowski J, Parisi MJ, Schultz CJ, Londos C, et al. Functional conservation for lipid storage droplet association among Perilipin, ADRP, and TIP47 (PAT)-related proteins in mammals, Drosophila, and Dictyostelium. J Biol Chem 2002;277:32253-7. doi:10.1074/jbc.M204410200.

[28] Wolins NE, Rubin B, Brasaemle DL. TIP47 associates with lipid droplets. J Biol Chem 2001;276:5101-8. doi:10.1074/jbc.M006775200.

[29] Robenek H, Hofnagel O, Buers I, Lorkowski S, Schnoor M, Robenek MJ, et al. Butyrophilin controls milk fat globule secretion. Proc Natl Acad Sci U S A 2006;103:10385-90. doi:10.1073/pnas.0600795103.

[30] Rylander L, Nilsson-Ehle P, Hagmar L. A simplified precise method for adjusting serum levels of persistent organohalogen pollutants to total serum lipids. Chemosphere 2006;62:333-6. doi:10.1016/j.chemosphere.2005.04.107.

[31] Richmond HD. THE RÖSE-GOTTLIEB METHOD OF MILK ANALYSIS. The Lancet 1927;209:1107. doi:10.1016/S0140-6736(00)75802-8. 